\title{
A Rare Case of Primary Angiosarcoma of Ovary in a Postmenopausal Woman
}

\author{
Kazila Bhutia ${ }^{\mathrm{a}, \mathrm{e}}$, Jun Jie Wang ${ }^{\mathrm{a}}$, Shahul Hameed Mohamed Siraj ${ }^{\mathrm{b}}$, Wai Kheong Ryan Lee ${ }^{\mathrm{a}}$, \\ May Ying Leong ${ }^{\mathrm{c}}$, Yong Kuei Lim ${ }^{\mathrm{d}}$
}

\begin{abstract}
Primary angiosarcoma of the ovary is a rare and aggressive subset accounting for less than $1 \%$ of ovarian malignancies. We present a rare case of primary angiosarcoma of the ovary in a 52-year-old healthy postmenopausal woman who presented with mild abdominal pain, distension and poor appetite of 1 week duration. On abdominal examination, right iliac fossa fullness with shifting dullness was noted. A CT of abdomen and pelvis revealed a right $10.5 \mathrm{~cm}$ complex solid cystic mass with ascites and peritoneal nodularity. Her CA125 was $560 \mathrm{U} / \mathrm{mL}$. She subsequently underwent full surgical staging and optimal debulking surgery. Final histology confirmed angiosarcoma of ovary grade 3 FIGO stage IIA ovarian cancer. A repeat CT of abdomen and pelvis done 4 weeks later prior to starting chemotherapy showed large $6.5 \times 6 \mathrm{~cm}$ pelvic peritoneal deposits with cystic septation and solid components. Her disease progressed despite palliative chemotherapy and she unfortunately passed away several months after surgery. We reiterate that primary angiosarcoma of the ovary is an aggressive tumor with poor prognosis despite optimal surgical treatment.
\end{abstract}

Keywords: Angiosarcoma; Cancer; Ovary

\section{Introduction}

Gynecological sarcomas are rare entities of which primary angiosarcoma of the ovary accounts for less than $1 \%$ of ovarian malignancies $[1,2]$. Primary angiosarcoma of the ovary has an overall poor prognosis with average survival rates ranging from 1 to 30 months [2,3]. The majority of cases reported are

\footnotetext{
Manuscript accepted for publication August 11, 2016

aDivision of Obstetrics \& Gynecology, KK Women's and Children's Hospital, Singapore

bDepartment of Obstetrics \& Gynecology, KK Women's and Children's Hospital, Singapore

'Department of Pathology, KK Women's and Children's Hospital, Singapore dDepartment of Gynaeoncology, KK Women's and Children's Hospital, Singapore

${ }^{\text {e}}$ Corresponding Author: Kazila Bhutia, Division of Obstetrics \& Gynecology, KK Women's and Children's Hospital, 100 Bukit Timah Road, Singapore 229899, Singapore. Email: Kazila.Bhutia@kkh.com.sg
}

doi: http://dx.doi.org/10.14740/jmc2606w in the premenopausal age group, with several cases reported in postmenopausal patients [2-7]. Here we report a rare case of a 52-year-old postmenopausal woman with primary ovarian angiosarcoma with an unusual clinical presentation of tachycardia with hemoperitoneum and immediate rapid progression of disease despite optimal debulking surgery.

\section{Case Report}

A 52-year-old, parity 4, postmenopausal woman with no significant past medical or surgical history was seen at a $24-\mathrm{h}$ women clinic in a tertiary hospital. She was referred by her general practitioner with mild abdominal pain and distension, and poor appetite lasting 1 week. There was no loss of weight, bowel or bladder complaint and she denied any vaginal bleed. On abdominal examination, fullness at the right iliac fossa with shifting dullness was noted. There was no tenderness, guarding or rigidity. On speculum examination, the cervix and vagina appeared normal. Pap smear was normal. Endometrial biopsy was negative for hyperplasia or carcinoma. Trans-abdominal pelvic ultrasound showed $11.8 \times 9.4 \times 8.8 \mathrm{~cm}$ heterogeneous mass in the right adnexal region. Bilateral ovaries were not seen separately and there was ascites in the pelvis. A subsequent CT scan of chest, abdomen and pelvis revealed a complex solid cystic mass measuring $10.3 \times 10.8 \mathrm{~cm}$ with enhancing solid component inseparable from uterus extending into right adnexal region (Fig. 1). There was no evidence of

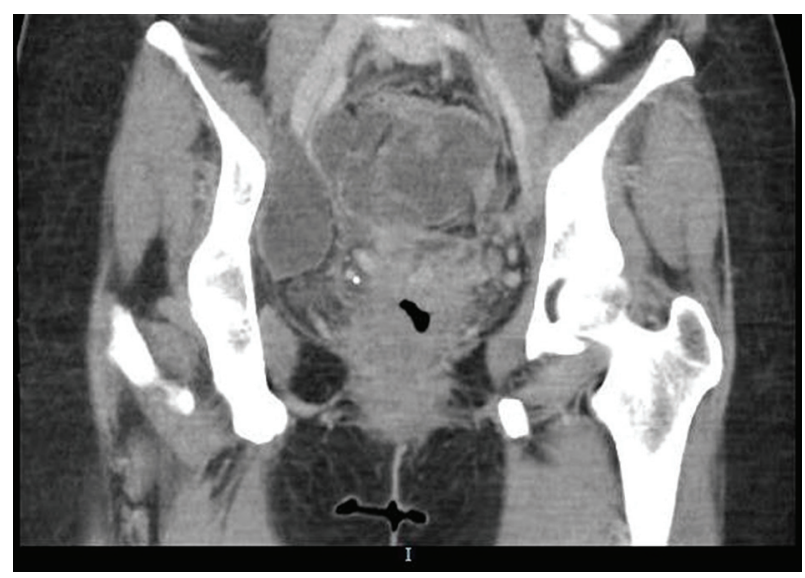

Figure 1. Complex solid cystic mass measuring $10.3 \times 10.8 \mathrm{~cm}$ with enhancing solid component inseparable from uterus extending into right adnexal region. 


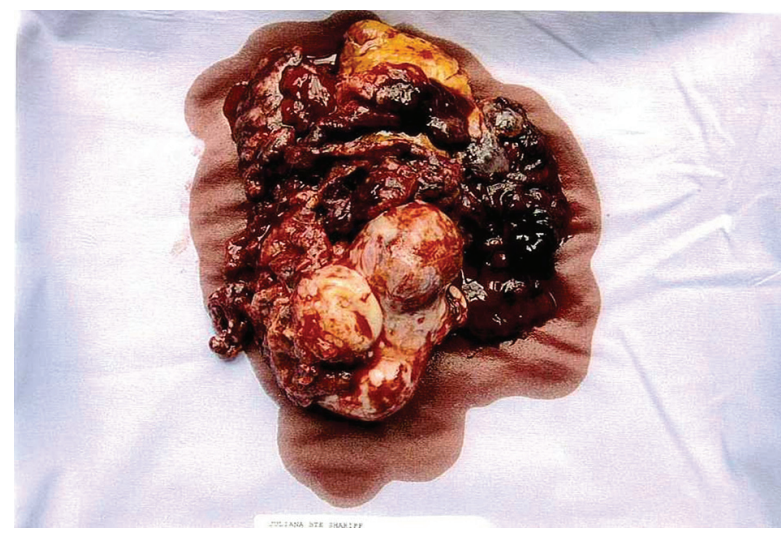

Figure 2. FIGO stage IIA grade 3 angiosarcoma of ovary.

enlarged abdominal or pelvic lymph nodes. Serum CA125 was elevated at $560 \mathrm{U} / \mathrm{mL}$.

In view of an advanced ovarian tumor, she was counseled for cytoreductive surgery which included a total abdominal hysterectomy, bilateral salphingoophorectomy, and infragastric omentectomy with bilateral pelvic and para-aortic lymphadenectomy. On the day of surgical admission, baseline tachycardia was noted with baseline heart rate ranging from 106 to $120 / \mathrm{min}$. Her pre-operative hemoglobin was $12.50 \mathrm{~g} / \mathrm{dL}$. Subsequently, she underwent immediate exploratory laparotomy where $3.5 \mathrm{~L}$ of hemoperitoneum with ascitic fluid was drained. There were bilateral ovarian tumors adherent to the pouch of Douglas and local invasion to back of uterus. Frozen section of pathological specimens of both ovaries revealed high grade malignant tumors. The uterus was normal in size, and liver and omentum were grossly normal. No enlarged lymph nodes were found. Optimal surgical debulking was achieved with no gross residual tumor seen at completion of surgery. Post-operative hemoglobin dropped from 12.5 to $8.5 \mathrm{~g} / \mathrm{dL}$ on post-operative day 3. She was discharged well 8 days after the operation.

The final histological result confirmed angiosarcoma of ovary with grade FIGO stage IIA ovarian cancer (Fig. 2). The tumor was identified in the uterine serosa and posterior paracervical tissue. There was no involvement of omentum and lymph nodes. She was referred to the sarcoma group (medical oncology) after a gyneoncology multi-disciplinary team meeting and offered adjuvant palliative chemotherapy.

Unfortunately, her cancer recurred very soon after surgery. A repeat $\mathrm{CT}$ of abdomen and pelvis done 4 weeks after surgery as a baseline scan prior to starting chemotherapy showed large $6.5 \times 6 \mathrm{~cm}$ pelvic peritoneal deposits with cystic septation and solid component (Fig. 3). The mass effect of the deposits caused compression of distal right ureter with mild hydronephrosis. Subsequently, she received two cycles of doxorubicin at 3 weeks interval. Consequently, she was admitted with hypokalemia, neutropenia sepsis and acute kidney insult 3 weeks after the second cycle of chemotherapy. Repeat CT scan of abdomen and pelvis showed interval increase in large pelvic mass $11 \times 12 \times 12 \mathrm{~cm}$ inseparable from the rectum and sigmoid colon causing right moderate hydronephrosis. There were also multiple peritoneal nodules with ascites. She underwent insertion of right nephrostomy by the intervention radiol-

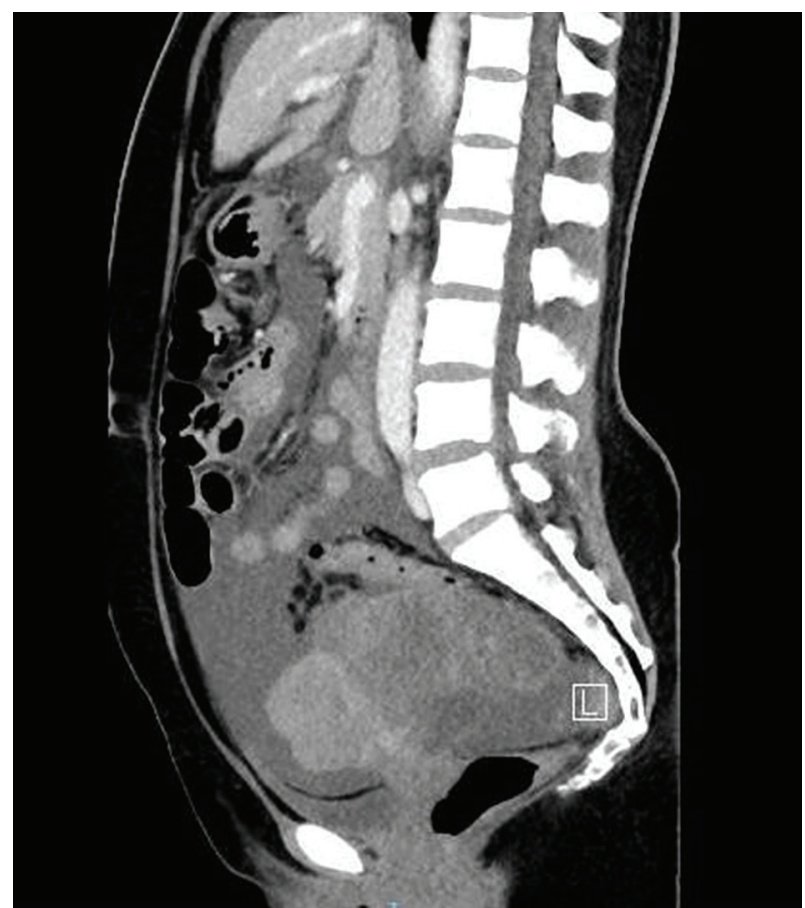

Figure 3. A large $6.5 \times 6 \mathrm{~cm}$ pelvic peritoneal deposits with cystic septations and solid components.

ogist and was discharged 1 week later. However, she was later readmitted within 2 weeks with abdominal pain secondary to the aggressively growing tumor mass and ascites. Given the rapid progression of disease within a short interval, the patient and her family opted for best supportive care. She was placed under the care of palliative team with regular analgesia and ascites drainage. She deteriorated rapidly clinically and passed away 5 months after surgery.

\section{Discussion}

Ovarian angiosarcoma is an extremely rare subset of ovarian tumor, with few cases reported so far. It is seen in all age groups, predominantly in premenopausal age, with only five cases reported so far in the postmenopausal age group [2-7]. Most of the cases reported are pure angiosarcoma in premenopausal patients. Among the five postmenopausal patients, two had concurrent mucinous cyst adenoma, one had fibroma and one had clear cell carcinoma and mature teratoma. In our case, the patient had a pure angiosarcoma of the ovary without other concurrent pathological tumors.

Most of these cases reported had advanced stage of disease at the time of presentation. They are associated with poor prognosis with most patients not surviving more than 30 months after initial diagnosis [2, 8-10]. In particular, patients with advanced disease FIGO stage III and above died from the disease in less than 9 months which was similar in our case [2].

The optimal treatment for advanced stage disease is full clinical surgical staging and adjuvant chemotherapy. However, angiosarcoma of an ovarian malignancy responds poorly 
to adjuvant chemotherapy. Chemotherapy should be considered as palliative rather than curative treatment in patients with advance disease. The most commonly used chemotherapy is doxorubicin and/or ifosfamide. Doxorubicin is the most active single agent chemotherapy of soft tissue sarcoma with overall response rate of $20 \%$ [11]. In our case, the patient received two cycles of doxorubicin at 3 weeks interval. Few cases reported the use of a combination of mesna, doxorubicin, ifosfamide and dacarbazine (MAID regimen) $[12,13]$. However, this regimen was associated with significant morbidity due to myelotoxicity and sepsis. In addition, gemcitabine and cisplatin were used in one case report with primary ovarian angiosarcoma and possible bone metastasis with MYC gene amplification and achieved progression free survival of at least 7 years [3].

Cancer staging appears to be the single most important prognostic parameter to determine the length of survival or disease free interval. The patient with FIGO stage I disease was reported to have disease free interval up to 9 years [8]. Stage IIA disease in an 11-year-old girl was reported to have disease free interval of 10 months [14]. Only about $40 \%$ of patients with FIGO stage III/IV have demonstrated the survival more than 6 months. Longest survival reported in literature was 29 months in stage IV $[2,10]$. In our case, the patient survived for 5 and 6 months after diagnosis and surgery, respectively. Unfortunately, she developed rapid advancement of disease after surgery which she succumbed to despite palliative chemotherapy.

In summary, this case highlights the rapid recurrence and overall poor prognosis of angiosarcoma of the ovary despite optimal surgical debulking surgery. The aggressive nature of this subset of ovarian cancer warrants further research where a more optimal chemotherapy regime may provide longer disease free survival and improve the overall prognosis in patients with advanced ovarian cancer.

\section{References}

1. Yaqoob N, Nemenqani D, Khoja H, Hafez M, Tulbah A, Al-Dayel F. Ovarian angiosarcoma: a case report and review of the literature. J Med Case Rep. 2014;8:47.

2. Yonezawa I, Waki M, Tamura Y, Onoda R, Narushima M, Ishizuka T, Tajima S. Gemcitabine-based regimen for primary ovarian angiosarcoma with MYC amplification. Curr Oncol. 2014;21(6):e782-789.
3. Bradford L, Swartz K, Rose S. Primary angiosarcoma of the ovary complicated by hemoperitoneum: a case report and review of the literature. Arch Gynecol Obstet. 2010;281(1):145-150.

4. Ongkasuwan C, Taylor JE, Tang CK, Prempree T. Angiosarcomas of the uterus and ovary: clinicopathologic report. Cancer. 1982;49(7):1469-1475.

5. Cambruzzi E, Pegas KL, Milani DM, Cruz RP, Guerra EH, Ferrari MB. Angiosarcoma arising in an ovarian fibroma: a case report. Patholog Res Int. 2010;2010:842592.

6. Bosmuller H, Gruber C, Haitchi-Petnehazy S, Wagner D, Webersinke G, Hauptmann S. Primary angiosarcoma of the ovary with prominent fibrosis of the ovarian stroma. Case report of an 81-year old patient. Diagn Pathol. 2011;6:65.

7. Takahashi H, Chaopotong P, Kajita S, Hashimura M, Yamazaki H, Saegusa M. Mixed angiosarcoma, clear cell adenocarcinoma and mature teratoma elements in an ovarian tumor: a case report and literature review. Pathol Int. 2012;62(8):538-542.

8. Nielsen GP, Young RH, Prat J, Scully RE. Primary angiosarcoma of the ovary: a report of seven cases and review of the literature. Int J Gynecol Pathol. 1997;16(4):378382 .

9. den Bakker MA, Ansink AC, Ewing-Graham PC. "Cutaneous-type" angiosarcoma arising in a mature cystic teratoma of the ovary. J Clin Pathol. 2006;59(6):658-660.

10. Contreras AL, Malpica A. Angiosarcoma arising in mature cystic teratoma of the ovary: a case report and review of the literature. Int J Gynecol Pathol. 2009;28(5):453457.

11. Cunningham MJ, Brooks JS, Noumoff JS. Treatment of primary ovarian angiosarcoma with ifosfamide and doxorubicin. Gynecol Oncol. 1994;53(2):265-268.

12. Platt JS, Rogers SJ, Flynn EA, Taylor RR. Primary angiosarcoma of the ovary: a case report and review of the literature. Gynecol Oncol. 1999;73(3):443-446.

13. Quesenberry CD, Li C, Chen AH, Zweizig SL, Ball HG, 3rd. Primary angiosarcoma of the ovary: a case report of Stage I disease. Gynecol Oncol. 2005;99(1):218-221.

14. Iljazovic E, Tomic S, Mustedanagic-Mujanovic J, Karasalihovic Z, Kuljanin M, Fatusic Z, Konjic E, et al. Angiosarcoma of the ovary in an 11 year old girl: case report and review of the literature. Bosn J Basic Med Sci. 2011;11(2):132-136. 